\title{
First-principles study of fcc-Ag/bcc-Fe interfaces
}

\author{
Song Lu, ${ }^{1, *}$ Qing-Miao Hu, ${ }^{2}$ Marko P. J. Punkkinen, ${ }^{3}$ Börje Johansson, ${ }^{1,4}$ and Levente Vitos ${ }^{1,4,5}$ \\ ${ }^{1}$ Applied Materials Physics, Department of Materials Science and Engineering, Royal Institute of Technology, Stockholm SE-100 44, Sweden \\ ${ }^{2}$ Shenyang National Laboratory for Materials Science, Institute of Metal Research, Chinese Academy of Sciences, \\ 72 Wenhua Road, Shenyang 110016, China \\ ${ }^{3}$ Department of Physics and Astronomy, University of Turku, FI-20014 Turku, Finland \\ ${ }^{4}$ Department of Physics and Astronomy, Division of Materials Theory, Uppsala University, Box 516, SE-751210, Uppsala, Sweden \\ ${ }^{5}$ Wigner Research Centre for Physics, Institute for Solid State Physics and Optics, H-1525 Budapest, P.O. Box 49, Hungary
}

(Received 11 April 2013; published 10 June 2013)

\begin{abstract}
$A b$ initio calculations are employed to determine the lower and upper bounds of the interfacial energy and work of separation of a fcc- $\mathrm{Ag} / \mathrm{bcc}-\mathrm{Fe}$ interface. The strain-free interfacial energy of the coherent interface is taken as the lower bound and the interfacial energy of the commensurate incoherent interface as the upper bound of the interfacial energy of a realistic semicoherent interface. The latter is estimated by applying an averaging scheme based on the interfacial energies obtained for the coherent interfaces. Similar calculations are performed for determining the bounds of the work of separation. We justify the use of the averaging scheme by carrying out large supercell calculations for a semicoherent interface. For a Fe(110)/Ag(111) semicoherent interface, we show that taking either $\mathrm{Fe}$ or $\mathrm{Ag}$ as the underlying lattice, our averaging scheme can yield a reasonable estimation of the work of separation of the semicoherent interface. However, when taking Ag as the underlying lattice, the averaged interfacial energy of the semicoherent interface is significantly underestimated due to the magnetism. The structure and magnetism at the coherent and semicoherent interfaces are discussed.
\end{abstract}

DOI: 10.1103/PhysRevB.87.224104

PACS number(s): 75.50.Bb, 71.15.Nc, 81.05.Zx

\section{INTRODUCTION}

Heterophase interfaces between face-centered-cubic (fcc) and body-centered-cubic (bcc) phases exist in many important alloys, such as $\mathrm{Fe}-\mathrm{Cu}, \mathrm{Fe}-\mathrm{Ni}, \mathrm{Fe}-\mathrm{Ag}, \mathrm{Ni}-\mathrm{Cr}, \mathrm{Cu}-\mathrm{Cr}$, or duplex stainless steels. The interfacial properties are very important, for example, when studying the precipitate growth kinetics and interface strengthening or failure mechanisms. It has been shown that the interfacial energy is a key parameter determining the nucleation barrier and also the shapes of precipitates. ${ }^{1-3}$ However, the interfacial energy is often not experimentally accessible. Due to the lack of measured data, ab initio calculations have been extensively applied to determine the metal-ceramic interfacial energy. ${ }^{4}$ However, for the metallic $\mathrm{fcc} / \mathrm{bcc}$ interface theoretical studies are very limited.

Experimental work has shown that during nucleation and in the early stage of growth, precipitates usually match the matrix coherently, which minimizes the interfacial energy. ${ }^{5}$ They may lose coherency during continued growth when the elastic strain energy contribution to the total free energy of the system becomes dominant. Formation of line defects, such as misfit dislocations and structure ledges, within the interface relieves misfit stress at the expense of increasing interfacial energy. ${ }^{2}$ By introducing the structure ledges, the interfacial coherency can be significantly increased. ${ }^{6,7}$

The coherent interface can be investigated by methods based on density functional theory (DFT), however, without considering the lattice mismatch or lattice relaxation. To model a realistic interface with mismatch (semicoherent interface) requires very large supercells which are normally beyond the capacities of nowadays DFT methods.

The interface properties of a semicoherent interface, such as interfacial energy or work of separation, may be divided into two parts: the properties of the ideal coherent interface (chemical contribution) and a correction term representing the contribution from the misfit dislocations or structure ledges (structure contribution). ${ }^{8,9}$ Some hybrid methods have been proposed to study the semicoherent interfaces. ${ }^{8-11}$ First, DFT calculations are performed to calculate the properties of the coherent interface. Then, based on the DFT results, embedded-atom methods (EAM) or continuum theory are adopted to evaluate the correction term induced by the presence of misfit dislocations. Using this methodology, various interfaces between metal and carbide, oxide, or intermetallics have been extensively investigated. For example, for the $\mathrm{NiAl} / \mathrm{Mo}$ interface with $\sim 9 \%$ mismatch, using a simple equation from the continuum theory, the presence of the misfit dislocation arrays was estimated to reduce the work of separation of the coherent interface from DFT calculations by about $10 \%,{ }^{12}$ which may, however, depend on the characteristics of the misfit dislocations. Based on $a b$ initio calculations, Medvedeva et al. ${ }^{13}$ showed that the misfit dislocation within the Peierls-Nabarro model ${ }^{10,11}$ may decrease the work of separation of the NiAl/Mo interface by $\sim 35 \%$. Since the error introduced by the continuum theory is difficult to estimate, it is important to define the upper and lower bounds of the excess energy of realistic semicoherent interfaces.

The embedded-atom method may seem to be an optimal choice to perform large-scale atomistic simulations for interfaces. ${ }^{14-17}$ However, a major impediment to those applications is the lack of reliable interatomic potentials for many materials. It is further argued that the EAM potentials are less reliable for bcc metals in which the interatomic forces include an angle-dependent component, compared to those for fcc metals. ${ }^{18,19}$ A good and consistent agreement in the description of the coherent interfaces (e.g., work of separation 
and interfacial energy) using the EAM potentials and DFT calculations would add more reliability for further applications of the EAM potentials to semicoherent or incoherent interfaces. ${ }^{15,20}$

In the present work, without trying to establish the exact physical parameters for semicoherent interfaces containing line defects, we perform first-principles calculations of the coherent and semicoherent interfaces to determine the work of separation and the excess interfacial energy. The information for the coherent interface itself is very important. For example, first-principles interfacial energy can be incorporated into the phase-field simulations to provide quantitative results which are comparable with experimental data (i.e., the equilibrium morphology of precipitate). ${ }^{21}$ Starting from the results obtained for the coherent interfaces, we estimate the work of separation and interfacial energy of the commensurate incoherent interface by an averaging scheme., ${ }^{4,22,23}$ The formation energies for the coherent and commensurate interfaces represent the lower and upper bounds for the semicoherent interfaces, respectively, which may be used to assess results based on the EAM potentials or continuum methods.

Here we consider the Fe/Ag interface as a model interface. Due to the immiscibility between $\mathrm{Fe}$ and $\mathrm{Ag}$, the $\mathrm{Fe} / \mathrm{Ag}$ interfaces exist in various Fe-Ag systems, for example, Fe/Ag multilayers and $\mathrm{Fe} / \mathrm{Ag}$ core/shell nanoparticles. The Fe/Ag multilayers show giant magnetoresistance and perpendicular magnetic anisotropy and have been extensively studied for many years. ${ }^{24}$ Recently, there is increasing interest in the $\mathrm{Fe} / \mathrm{Ag}$ core/shell nanoparticles possessing unusual magnetic and optical properties with potential applications. ${ }^{25,26}$ The knowledge of the interfacial energy is important for the nanoparticle growth. In the present work, two types of Fe/Ag shape interface are considered, the $\mathrm{Fe}(001) / \mathrm{Ag}(001)$ and the $\mathrm{Fe}(110) / \mathrm{Ag}(111)$ interfaces. However, we would like to emphasize the methodology we develop here to define the upper and lower bounds of the interfacial energy and work of separation of the semicoherent interfaces. The proposed approach may be generalized and applied to other metallic $\mathrm{fcc} / \mathrm{bcc}$ interfaces.

The rest of this paper is arranged as follows: We describe the computational models of the two types of interfaces and the calculation details in Sec. II. The results are presented and discussed in Sec. III. In Sec. III A we present the calculated equilibrium surface energies. Our results for the work of separation and interfacial energy for the coherent $\mathrm{Fe}(001) / \mathrm{Ag}(001)$ and $\mathrm{Fe}(110) / \mathrm{Ag}(111)$ interfaces are presented in Sec. III B. Then we introduce an averaging scheme for estimating the work of separation and interfacial energy of a semicoherent/incoherent $\mathrm{Fe}(110) / \mathrm{Ag}(111)$ interface in Sec. III C. Interface structure and magnetism are discussed in Sec. III D. The main findings are summarized in Sec. IV.

\section{METHODOLOGY}

\section{A. Model for the $\mathrm{Fe}(001) / \mathrm{Ag}(001)$ coherent interface}

Experimentally it is found that the Fe film grows on the $\operatorname{Ag}(001)$ surface as a bcc $\alpha$-Fe film rotated by $45^{\circ}$ relative to the underlying lattice. ${ }^{27,28}$ The interface between $\mathrm{Fe}$ and $\mathrm{Ag}$ has the orientation relationship of $\mathrm{Fe}(100) \| \mathrm{Ag}(100)$ and
$[100]_{\mathrm{Ag}} \|[1 \overline{1} 0]_{\mathrm{Fe}}$. Similar cube-on-cube interface was also reported for the $\mathrm{Fe}(001) / \mathrm{Au}(001)$ interface in the $\mathrm{Fe} / \mathrm{Au}$ core/shell nanoparticles by Benoit. ${ }^{29}$ The $\mathrm{Fe}(001) / \mathrm{Ag}(001)$ interface is modeled by a supercell containing $N$ layers of $\mathrm{Fe}(001)$ plane and 11 layers of $\mathrm{Ag}(001)$ plane using periodic boundary conditions. The Fe-Ag slabs are separated by a vacuum of thickness larger than $10 \AA$. A schematic sketch of the supercell is shown in Fig. 1(a). The number of layers for both $\mathrm{Fe}$ and $\mathrm{Ag}$ is carefully tested to reach good convergence, which will be presented in Sec. III B. We take Ag as the matrix of the system and the Fe lattice is distorted laterally to match the lattice of $\mathrm{Ag}$ substrate so as to achieve coherency. All atoms are allowed to relax in the direction perpendicular to the interface. The mismatch between $\mathrm{Fe}(001)$ and $\mathrm{Ag}(001)$ along the $[110]_{\mathrm{Ag}}$ direction is defined as

$$
\epsilon=\left(\sqrt{2} a_{\mathrm{Fe}}-a_{\mathrm{Ag}}\right) / a_{\mathrm{Ag}},
$$

where $a_{\mathrm{Fe}}$ and $a_{\mathrm{Ag}}$ are the equilibrium lattice parameters of $\mathrm{Fe}$ and $\mathrm{Ag}$. In the present work we calculate all mismatches relative to the underlying lattice. There are several high symmetry sites on the $\operatorname{Ag}(001)$ plane for the successive $\mathrm{Fe}$ atom to form the interface. These are the bcc site, bridge site, and top site, as depicted in Fig. 1(a). However, in the present study for the $\mathrm{Fe}(001) / \mathrm{Ag}(001)$ interface, only the most stable coherent interface with interface $\mathrm{Fe}$ atoms sitting at the bcc sites is considered.

\section{B. Model for the $\mathrm{Fe}(110) / \mathrm{Ag}(111)$ coherent interface}

In the thin film of Fe/Ag multilayers, it was reported that the interface is atomically sharp and follows the NishiyamaWasserman (NW) orientation relationship. ${ }^{30}$ As a first step to investigate the complicated $\mathrm{Fe}(110) / \mathrm{Ag}(111)$ semicoherent interface, we start from the coherent $\mathrm{Fe}(110) / \mathrm{Ag}(111)$ interface with the NW orientation relationship $[1 \overline{10}]_{\mathrm{Ag}} \|[001]_{\mathrm{Fe}}$. The interface is modeled by a supercell composed of nine layers of $\mathrm{Ag}(111)$ and $N$ layers of $\mathrm{Fe}(110)$. The thickness of vacuum separating the $\mathrm{Fe}(110) / \mathrm{Ag}(111)$ slabs is set to more than $10 \AA$. Each layer has only one atom. The Fe lattice is laterally strained to fit the underlying $\operatorname{Ag}(111)$ lattice, namely, the Fe lattice is stretched by around $26.8 \%$ along the $[110]_{\mathrm{Fe}}$ direction and by about $3.4 \%$ along the $[001]_{\mathrm{Fe}}$ direction. Along the direction perpendicular to the interface, the Fe lattice keeps the bcc stacking sequence abababa... and the Ag lattice keeps the fcc stacking sequence $A B C A B C A B C \ldots$... For the coherent $\mathrm{Fe}(110) / \mathrm{Ag}(111)$ interface, we have the freedom to shift the $\mathrm{Fe}$ lattice against the Ag lattice on the interface plane. There are three inequivalent parallel translation states for the successive Fe atom at the interface, corresponding to three high-symmetry positions relative to the (111)Ag plane. They are termed as the fcc site, bridge site, and top site, ${ }^{22,23}$ as marked in Fig. 1(b). The convergence of the work of separation and interfacial energy with respect to the number of Fe layers was tested for these translation states, and results are presented in Sec. III B.

\section{Work of separation and interfacial energy}

The work of separation $(W)$ is defined as the energy required per unit area to reversibly separate a bulk material into two semi-infinite bulks with two free surfaces. It is sometimes also 


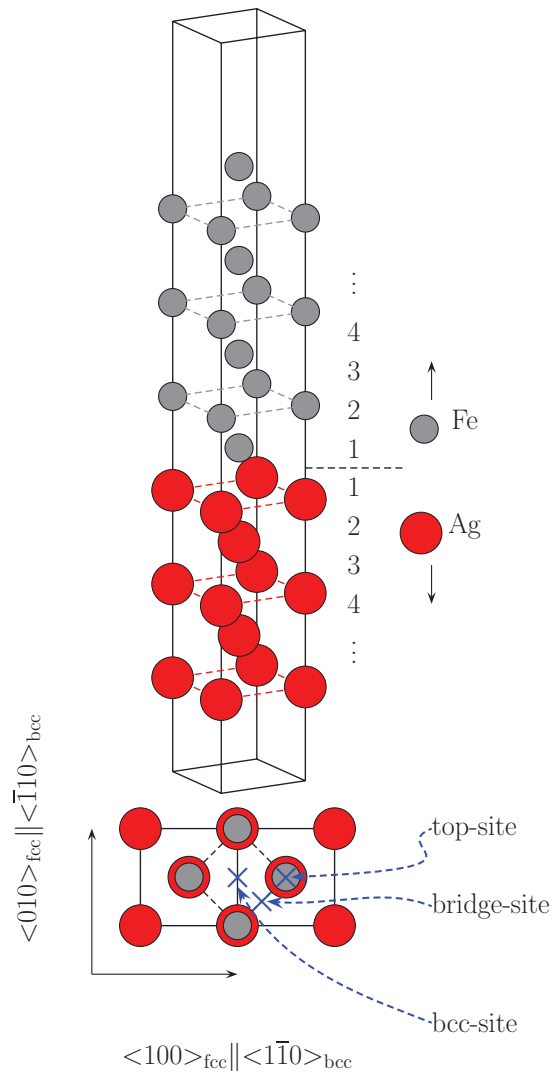

(a)

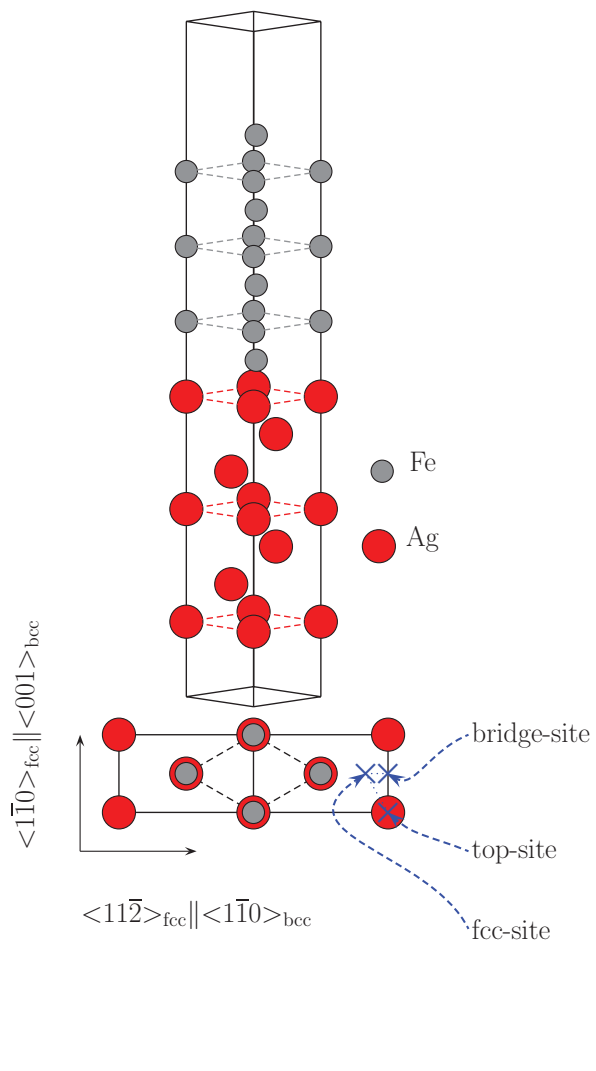

(b)

FIG. 1. (Color online) Schematic of the (a) $\mathrm{Fe}(001) / \mathrm{Ag}(001)$ interface and the (b) $\mathrm{Fe}(110) / \mathrm{Ag}(111)$ interface. The cross symbols mark the high-symmetry sites with respect to the Ag plane for the successive Fe atom at interface. The layers around the interface are indexed by numbers.

named as the "ideal work of separation."22,29 In the present study, $W$ is calculated according to

$$
W=\left[E_{\mathrm{Fe}}+E_{\mathrm{Ag}}-E_{\mathrm{Fe} / \mathrm{Ag}}\right] / A,
$$

where $E_{\mathrm{Fe}}$ and $E_{\mathrm{Ag}}$ are the total energies of the Fe and Ag slabs with free surfaces, respectively, $E_{\mathrm{Fe} / \mathrm{Ag}}$ is the total energy of the $\mathrm{Fe} / \mathrm{Ag}$ supercell slab embedded in vacuum, and $A$ is the area of the interface. All systems were calculated under exactly the same conditions ( $k$ mesh, cutoff energy, etc.). They were all subject to the same lateral lattice strain set by the underlying lattice. Perpendicular to the interface, all the atoms were fully relaxed. The work of separation calculated in this manner gives direct information regarding the strength and bonding of the interface, ${ }^{22}$ and was taken as a measure for the mechanical stability and chemical bonding at the interface. ${ }^{31}$

The interfacial energy $\gamma$ is calculated as

$$
\gamma=\left(E_{\mathrm{Fe} / \mathrm{Ag}}^{\prime}-E_{\mathrm{Fe}}^{\mathrm{Bulk}}-E_{\mathrm{Ag}}^{\mathrm{Bulk}}\right) / A,
$$

where $E_{\mathrm{Fe} / \mathrm{Ag}}^{\prime}=E_{\mathrm{Fe} / \mathrm{Ag}}-\sigma_{\mathrm{Fe}} A-\sigma_{\mathrm{Ag}} A$ is total energy of the $\mathrm{Fe} / \mathrm{Ag}$ supercell corrected for the free surfaces of the Fe and $\mathrm{Ag}$ parts of the Fe/Ag supercell slab embedded in vacuum. $E_{\mathrm{Fe}}^{\mathrm{Bulk}}, E_{\mathrm{Ag}}^{\mathrm{Bulk}}$ correspond to the total energies of $\mathrm{Fe}$ and $\mathrm{Ag}$ in bulk states, and $\sigma_{\mathrm{Fe}}$ and $\sigma_{\mathrm{Ag}}$ are the surface energies of the free surfaces of the Fe and Ag slabs, respectively. All systems were subject to the same lateral strain imposed by the underlying lattice. The surface energies are also calculated relative to the bulk suffering the same strain as the interface,

$$
\sigma_{\mathrm{X}}=\left(E_{\mathrm{X}}-E_{\mathrm{X}}^{\mathrm{Bulk}}\right) / 2 A,
$$

where X stands for Fe or Ag. Combining Eqs. (2), (3), and (4), the interfacial energy can also be expressed as

$$
\gamma=\sigma_{\mathrm{Fe}}+\sigma_{\mathrm{Ag}}-W .
$$

Equation (5) presents the relationship between $W$ and $\gamma$, and expresses the fact that the interfacial energy varies essentially opposite to the work of separation. ${ }^{23}$

\section{Density functional theory method}

The density functional theory ${ }^{32}$ calculations were performed by using the Vienna $a b$ initio simulation package (VASP) ${ }^{33-35}$ employing the Perdew-Burke-Ernzerhof $(\mathrm{PBE})^{36}$ generalized gradient approximation (GGA) for the exchange and correlation energy. The projector augmentedwave $(\mathrm{PAW})^{37}$ method was applied. For both bulk and interface calculations the cutoff energies were set to $400 \mathrm{eV}$ for both $\mathrm{Fe}$ and Ag. For the bulk calculations, a Monkhorst-Pack mesh of $21 \times 21 \times 21 k$ points was employed to sample the Brillouin zone. However, the $\Gamma$-centered grids of $21 \times 21 \times 1$ were adopted for the interface and the surface calculations. A $k$ mesh of $3 \times 9 \times 1$ was used for the calculations of the semicoherent 
interface. Spin-polarized calculations were carried out for all systems containing Fe atoms.

\section{RESULTS AND DISCUSSION}

\section{A. Equilibrium surface energy}

The calculated surface energies of the equilibrium $\mathrm{Fe}$ and Ag surfaces are calculated and summarized in Table I, together with other theoretical and experimental results. The presently calculated surface energies for $\mathrm{Ag}(001)$ and $\mathrm{Ag}(111)$ surfaces are $\sigma_{\mathrm{Ag}}^{001}=0.81 \mathrm{~J} \mathrm{~m}^{-2}$ and $\sigma_{\mathrm{Ag}}^{111}=0.68 \mathrm{~J} \mathrm{~m}^{-2}$, respectively, which agrees well with former pseudopotential (PP) calculations (GGA). ${ }^{38,39}$ GGA leads to smaller surface energy compared to the local density approximation (LDA) and estimated ("experimental") results. ${ }^{38}$ The calculated surface energies of the $\mathrm{Fe}(001)$ and the $\mathrm{Fe}(110)$ surfaces are $\sigma_{\mathrm{Fe}}^{001}=$ $2.54 \mathrm{~J} \mathrm{~m}^{-2}$ and $\sigma_{\mathrm{Fe}}^{110}=2.45 \mathrm{~J} \mathrm{~m}^{-2}$, respectively, which also agrees with previous calculations. ${ }^{44}$ Note that the experimental surface energies were determined by extrapolating the surface data measured for the liquid phase down to $0 \mathrm{~K} .^{43}$

\section{B. The coherent interfaces}

\section{The $\mathrm{Fe}(100) / \mathrm{Ag}(100)$ interface}

Relative to the $\operatorname{Ag}(100)$ plane, the mismatch $\epsilon$ calculated with the experimental lattice constants $\left(a_{\mathrm{Ag}}=4.0862 \AA, a_{\mathrm{Fe}}=\right.$ $2.8665 \AA)^{28}$ is $\sim 0.8 \%$ in the $[110]_{\mathrm{Ag}}$ direction. The calculated (GGA) lattice constants for $\mathrm{Ag}$ and Fe are 4.147 and $2.833 \AA$, respectively, which yield somewhat larger theoretical lattice mismatch $(\sim 3.4 \%)$. The effect of a larger mismatch caused by the exchange-correlation functional (GGA) on the work of separation of the $\mathrm{Fe}(001) / \mathrm{Au}(001)$ interface was investigated by Benoit et al. ${ }^{29}$ They found that this effect causes about $4 \%$ error on the work of separation. For the $\mathrm{Fe}(001) / \mathrm{Ag}(001)$ interface, we expect similar errors.

The calculated work of separation $(W)$ and interfacial energy $(\gamma)$ with respect to the number of $\operatorname{Fe}(001)$ layers $(N)$ are presented in Fig. 2. The work of separation and interfacial energy reach convergence at around nine layers of $\mathrm{Fe}(001)$, which results in $W \approx 2.30 \mathrm{~J} \mathrm{~m}^{-2}$ and $\gamma \approx 0.89 \mathrm{~J} \mathrm{~m}^{-2}$. The surface energy of the $\mathrm{Fe}(001)$ surface which is subject to the lateral strain set by the $\mathrm{Ag}$ lattice is $\sim 2.38 \mathrm{~J} \mathrm{~m}^{-2}$, i.e., $6.3 \%$

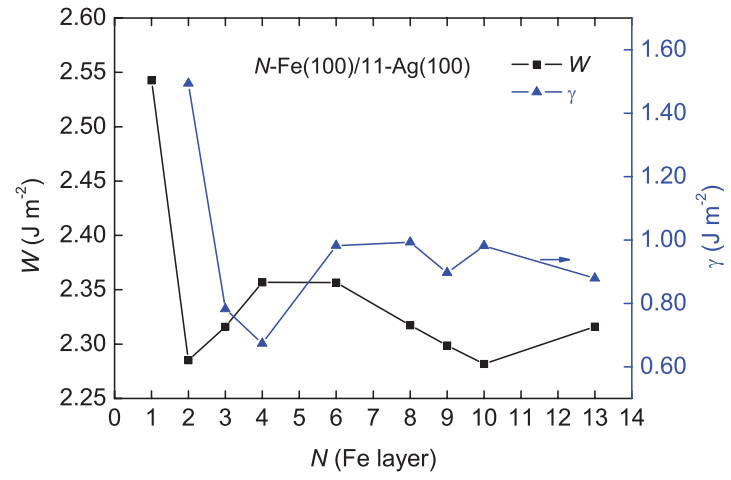

FIG. 2. (Color online) The calculated work of separation $(W)$ and interfacial energy $(\gamma)$ with respect to the number of $\mathrm{Fe}(001)$ layers for the $\mathrm{Fe}(001) / \mathrm{Ag}(001)$ interface. The lateral lattice constants of the supercell are fixed to those of $\operatorname{Ag}(001)$.

smaller than the value for the equilibrium $\mathrm{Fe}(001)$ surface (Table I).

The equilibrium interlayer distance between the $\operatorname{Ag}(001)$ layers $\left(d_{\mathrm{Ag}}^{001}\right)$ is $2.07 \AA$. Because of the Poisson effect, the interlayer distance between the strained bulklike $\mathrm{Fe}(001)$ layers $\left(d_{\mathrm{Fe}}^{001 \prime}\right)$ is around $1.38 \AA$, which is $\sim 2.5 \%$ smaller than the corresponding equilibrium interlayer distance for bcc Fe $\left(d_{\mathrm{Fe}}^{001} \approx 1.42 \AA\right)$. At the interface, the distance between $\mathrm{Ag}$ and Fe layers (interface separation, $d_{11}^{\mathrm{Fe}, \mathrm{Ag}}$ ) is $\sim 1.85 \AA$, which is $\sim 6 \%$ smaller than the averaged interlayer distance $\left[\left(d_{\mathrm{Ag}}^{001}+d_{\mathrm{Fe}}^{001}\right) / 2\right]$. Only the first interlayer distance between Fe layers $\left(d_{12}^{\mathrm{Fe}}\right)$ at the interface is markedly decreased by $\sim 6 \%$ compared to $d_{\mathrm{Fe}}^{001 \prime}$. Starting from the second-nearest layer around the interface, the interface has nearly no effect on the interlayer distance.

The magnetic moment of the $\mathrm{Fe}$ atom at the interface is remarkably enhanced $\left(\sim 2.82 \mu_{\mathrm{B}}\right)$, compared to that of the bulklike Fe atom $\left(\sim 2.40 \mu_{\mathrm{B}}\right)$. This value is only slightly smaller than the surface magnetic moment $2.99 \mu_{\mathrm{B}}$ for the laterally strained Fe surface. These findings agree well with the previous calculations. ${ }^{45,46}$ Generally speaking, the Fe atoms at the interface behave in a way similar to the atoms at the surface. The structure and electronic properties of a similar interface $\mathrm{Au}(001) / \mathrm{Fe}(001)$ was recently investigated by Benoit

TABLE I. Theoretical [present results, pseudopotential (PP), full-potential (FP)] surface energies for the equilibrium Ag and Fe surfaces, in units of $\mathrm{J} \mathrm{m}^{-2}$. For comparison, the estimated isotropic surface energies are also listed.

\begin{tabular}{lccccc}
\hline \hline Surface energy & Present & PP-GGA & PP-LDA & FP-LDA & Estimated \\
\hline $\operatorname{Ag}(001)$ & 0.81 & $0.80,{ }^{\mathrm{a}} 0.80^{\mathrm{b}}$ & $1.35^{\mathrm{a}}$ & $1.21,{ }^{\mathrm{c}} 1.27^{\mathrm{d}}$ & $0.88,{ }^{\mathrm{e}} 1.24,{ }^{\mathrm{f}} 0.55^{\mathrm{e}}$ \\
$\mathrm{Ag}(111)$ & 0.68 & $0.76,{ }^{\mathrm{a}} 0.58-0.62^{\mathrm{b}}$ & $1.13^{\mathrm{a}}$ & $1.21^{\mathrm{c}}$ & $-2.42-2.55^{\mathrm{h}}$ \\
$\mathrm{Fe}(001)$ & 2.54 & $2.48,{ }^{\mathrm{g}} 2.50^{\mathrm{h}}$ & - & - & - \\
$\mathrm{Fe}(110)$ & 2.45 & $2.42^{\mathrm{h}}$ & - & - \\
\hline \hline
\end{tabular}

\footnotetext{
${ }^{\text {a Reference } 38 .}$

${ }^{\mathrm{b}}$ Reference 39.

${ }^{\mathrm{c}}$ Reference 40

${ }^{\mathrm{d}}$ Reference 41 .

${ }^{\mathrm{e}}$ Reference 42.

${ }^{f}$ Reference 43.

${ }^{\mathrm{g}}$ Reference 29.

${ }^{\mathrm{h}}$ Reference 44.
} 


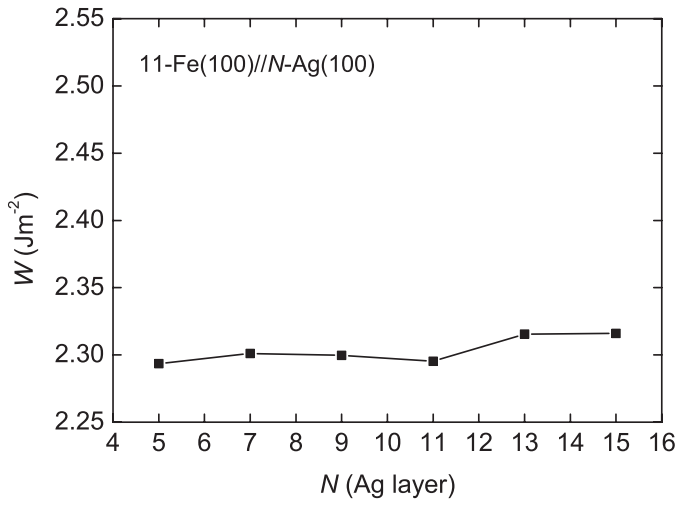

FIG. 3. The calculated work of separation with respect to the number of $\operatorname{Ag}(001)$ layers for the $\mathrm{Fe}(001) / \mathrm{Ag}(001)$ interface. Full relaxation is performed.

et al. ${ }^{29}$ using DFT calculations. For a detailed discussion about the interlayer distance and the magnetic moment, readers are referred to Ref. 29.

In order to study the lattice restraining effect on the calculated work of separation, we also calculated the work of separation with a fully relaxed supercell $11-\mathrm{Fe}(001) / N$ $\mathrm{Ag}(001)$ (without a vacuum layer). Note that the fully relaxed results depend on the size of the supercell. The results are shown in Fig. 3. The equilibrium in-plane lattice constant increases from 2.85 to $2.87 \AA$ with increasing the number of $\mathrm{Ag}$ layers from 5 to 15 , while the change in the work of separation is only about $0.4 \%$.

\section{The Fe(110)/Ag(111) coherent interface}

The calculated work of separation and interfacial energy for different stacking sequences with respect to the number of $\mathrm{Fe}(110)$ layers $(N)$ are shown in Figs. 4 and 5, respectively. We get converged results at about $N=7$. The corresponding work of separation and interfacial energy for different stacking sequences are $W_{\mathrm{fcc}}=2.21 \mathrm{~J} \mathrm{~m}^{-2}, W_{\text {bridge }}=2.04 \mathrm{~J} \mathrm{~m}^{-2}$, $W_{\text {top }}=1.43 \mathrm{~J} \mathrm{~m}^{-2}, \gamma_{\mathrm{fcc}}=0.36 \mathrm{~J} \mathrm{~m}^{-2}, \gamma_{\text {bridge }}=0.53 \mathrm{~J} \mathrm{~m}^{-2}$, and $\gamma_{\text {top }}=1.13 \mathrm{~J} \mathrm{~m}^{-2}$, respectively.

To investigate the effect of the lattice relaxation on the work of separation and interfacial energy, we also calculated

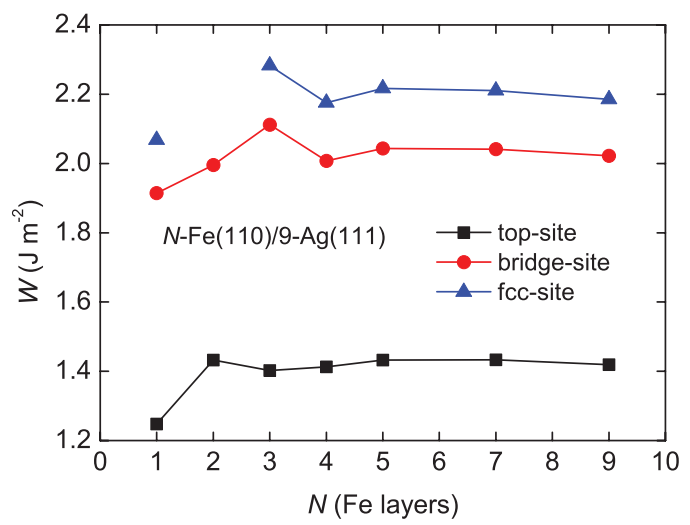

FIG. 4. (Color online) The calculated work of separation for the $N-\mathrm{Fe}(110) / 9-\mathrm{Ag}(111)$ interface for different stacking sequences with respect to $N$.

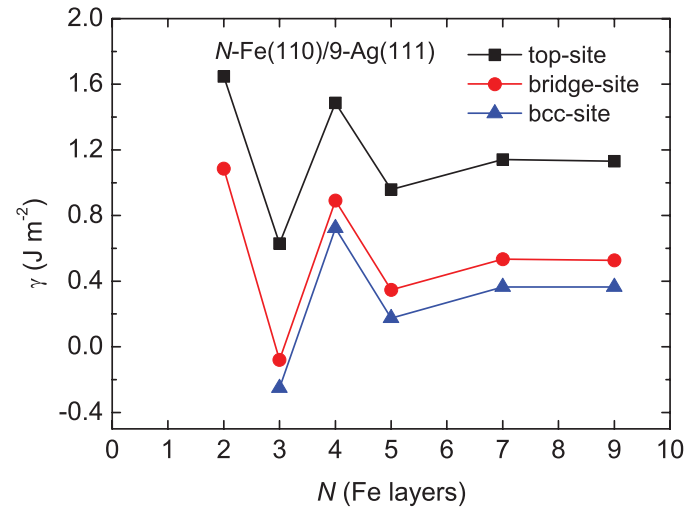

FIG. 5. (Color online) The calculated interfacial energy for the $N-\mathrm{Fe}(110) / 9-\mathrm{Ag}(111)$ interface for different stacking sequences with respect to $N$.

the work of separation and interfacial energy of the coherent $\mathrm{Fe}(110) / \mathrm{Ag}(111)$ interface with the in-plane lattice constants fixed to those of $\mathrm{Fe}(110)$ [taking $\mathrm{Fe}(110)$ as the underlying lattice]. These results are listed in Table II, along with those obtained when using $\operatorname{Ag}(111)$ as the underlying lattice. Notice that when taking bcc $\mathrm{Fe}$ as the underlying lattice, there is one more high-symmetry position which is termed as the bcc site. ${ }^{22}$ Because of the change in the in-plane symmetry, we cannot directly compare two sets of results. However, from Table II we find that the highest $W$ for both cases are very close to each other $\left(W_{\mathrm{fcc}}=2.21 \mathrm{~J} \mathrm{~m}^{-2}\right.$ for $\mathrm{Ag}$ as the underlying lattice versus $W_{\mathrm{bcc}}=2.24 \mathrm{~J} \mathrm{~m}^{-2}$ for $\mathrm{Fe}$ as the underlying lattice). The largest deviation is found for the case of the top site, where the difference is still less than 15\%. From these results, we may conclude that the work of separation is not very sensitive to lattice distortion within the presently studied range of distortion. Adham et al. ${ }^{15}$ compared the work of separation of the coherent bcc-Fe(110)/fcc-Ni(111) and fcc-Fe(111)/fcc-Ni(111) interfaces and found that $W$ is almost the same for the two interfaces. The same situation holds for the bcc-Fe(110)/bcc-Cr(110) and fcc-Fe(111)/bcc$\mathrm{Cr}(110)$ interfaces. ${ }^{15}$ The above results imply that the work of separation primarily gives information on the bonding at the interface. For a specific atomic site at a real interface, the local structure may deviate from both bcc or fcc, however, its contribution to the work of separation is expected to be close to the value calculated for a model coherent interface with the same translation state, no matter if taking fcc or bcc as the underlying lattice.

The surface energies of the strained and equilibrium surfaces are also listed in Table II. We see that expanding the Fe lattice to match the Ag lattice decreases the Fe(110) surface energy from 2.45 to $1.88 \mathrm{~J} \mathrm{~m}^{-2}$, whereas compressing the $\mathrm{Ag}$ lattice to match $\mathrm{Fe}$ decreases the $\mathrm{Ag}(111)$ surface energy from 0.68 to $0.51 \mathrm{~J} \mathrm{~m}^{-2}$. The difference in the surface energies of the equilibrium and strained surfaces results in a great difference in the calculated interfacial energies of the interfaces with $\mathrm{Fe}$ and Ag treated as substrates according to Eq. (5). For example, the smallest interfacial energy of the interface with Fe as substrate $\left(\gamma_{\mathrm{bcc}}=0.72 \mathrm{~J} \mathrm{~m}^{-2}\right)$ is twice the smallest interfacial energy of the one with $\mathrm{Ag}$ as substrate $\left(\gamma_{\mathrm{fcc}}=0.36 \mathrm{~J} \mathrm{~m}^{-2}\right)$. Therefore in this case, the coherent interfacial energy is very sensitive to the 
TABLE II. Theoretical work of separation $(W)$ and interfacial energy $(\gamma)$ for the coherent $\mathrm{Fe}(110) / \mathrm{Ag}(111)$ interface, taking the Fe(110) or the $\operatorname{Ag}(111)$ as the underlying lattice, respectively. Surface energies $(\sigma)$ for the equilibrium and strained states are also listed. All energies are expressed in $\mathrm{J} \mathrm{m}^{-2}$.

\begin{tabular}{|c|c|c|c|c|c|c|c|c|c|c|}
\hline $\begin{array}{l}\text { Underlying } \\
\text { lattice }\end{array}$ & $W_{\text {top }}$ & $W_{\text {fcc }}$ & $W_{\text {bridge }}$ & $W_{\text {bcc }}$ & $\sigma_{\mathrm{Ag}}$ & $\sigma_{\mathrm{Fe}}$ & $\gamma_{\text {top }}$ & $\gamma_{\mathrm{fcc}}$ & $\gamma_{\text {bridge }}$ & $\gamma_{b c c}$ \\
\hline $\mathrm{Fe}(110)$ & 1.64 & 2.18 & 2.02 & 2.24 & 0.51 & 2.45 & 1.32 & 0.79 & 0.94 & 0.72 \\
\hline $\operatorname{Ag}(111)$ & 1.43 & 2.21 & 2.04 & - & 0.68 & 1.88 & 1.13 & 0.36 & 0.53 & - \\
\hline
\end{tabular}

choice of the underlying lattice, unlike in the case of work of separation. We calculate the surface energy of the Fe(110) surface without spin polarization and find that the surface energy only decreases by $\sim 12 \%$ from $\sim 2.55$ to $\sim 2.24 \mathrm{~J} \mathrm{~m}^{-2}$ during distorting the Fe lattice to match the Ag lattice. We can see that the magnetism does not significantly affect the surface energy of $\mathrm{Fe}$ at the equilibrium states $\left(2.45 \mathrm{~J} \mathrm{~m}^{-2}\right.$ for magnetic state versus $2.55 \mathrm{~J} \mathrm{~m}^{-2}$ for nonmagnetic state), which agrees well previous study. ${ }^{44}$ However, for the strained state, the surface energy of $\mathrm{Fe}$ is remarkably decreased by the magnetism from 2.24 (nonmagnetic) to $1.88 \mathrm{~J} \mathrm{~m}^{-2}$ (ferromagnetic). Therefore, both the significant decrease of the Fe(110) surface energy and the sensitivity of the coherent interfacial energy to the underlying lattice originate from the magnetism.

Within a pair interaction model, Borchers and Bormann ${ }^{30}$ estimated the chemical contribution to the energy of coherent $\mathrm{Fe} / \mathrm{Ag}$ interfaces to be $\sim 0.88 \mathrm{~J} \mathrm{~m}^{-2}$, which is in line with our results. There is no experimental interfacial energy available for the solid-solid Fe/Ag interface. The interfacial energy of liquid $\mathrm{Fe} / \mathrm{Ag}$ interface at $1550{ }^{\circ} \mathrm{C}$ was estimated to be $1.04 \mathrm{~J} \mathrm{~m}^{-2}{ }^{47}$ Considering the positive temperature dependence of the interfacial energy, ${ }^{48}$ our results can be considered reasonable. In the literature, there are few data for the fcc/bcc interface. By analyzing the coarsening data with the LifshitzSlyozov-Wagner theory, Kita and Monzen ${ }^{49}$ estimated the interfacial energies of the incoherent $\alpha-\mathrm{Fe} / \mathrm{Cu}$ and coherent $\gamma-\mathrm{Fe} / \mathrm{Cu}$ interfaces to be 0.52 and $0.25 \mathrm{~J} \mathrm{~m}^{-2}$, respectively. First-principles study of the coherent $\mathrm{Cu}(111) / \mathrm{Ta}(110)$ interface (taking bcc Ta as the substrate) generated the interfacial energies of 0.12 and $0.45 \mathrm{~J} \mathrm{~m}^{-2}$ for the bcc-stacking and fcc-stacking interfaces, respectively. ${ }^{22}$ Comparing our results to the above data, though they are for different systems, indicates the same order of magnitude.

The work of separation $W_{\text {fcc }}$ of the most stable $\mathrm{Fe}(110) / \mathrm{Ag}(111)$ coherent interface $\left(2.21 \mathrm{~J} \mathrm{~m}^{-2}\right)$ is smaller than the one for the most stable $\mathrm{Fe}(001) / \mathrm{Ag}(001)$ coherent interface $\left(2.30 \mathrm{~J} \mathrm{~m}^{-2}\right)$. This is also the case for the interfacial energy $\left[0.36 \mathrm{~J} \mathrm{~m}^{-2}\right.$ for $\mathrm{Fe}(110) / \mathrm{Ag}(111)$ and $0.89 \mathrm{~J} \mathrm{~m}^{-2}$ for $\mathrm{Fe}(001) / \mathrm{Ag}(001)]$. These results follow the normal expectation, namely that the closed-packed interface should have smaller interfacial energy than the open interfaces.

\section{The semicoherent and incoherent $\mathrm{Fe}(110) / \mathrm{Ag}(111)$ interfaces}

In the following sections we introduce an averaging scheme which may be used to evaluate the work of separation and interfacial energies of the semicoherent/incoherent interface starting from the results obtained for the coherent interfaces. Direct calculations for a semicoherent interface are used to measure the reliability of the averaging scheme. Then the scheme is applied to study the incoherent Fe/Ag interface.

\section{The averaging scheme}

Based on pure DFT calculations, Benedek et $a l .{ }^{4}$ investigated the $\mathrm{MgO} / \mathrm{Cu}$ interface using a rather large supercell (399 atoms) to take into account the lattice constant mismatch and atomic relaxation at the interface. They showed that the properties of real equilibrium interfaces (e.g., work of separation) may be reproduced as weighted averages of the properties of coherent interfaces calculated for translation states with high symmetry (top site, fcc site, bridge site, etc.). EAM study also showed that the elastic contribution to the interfacial energy is negligible for large lattice mismatch, ${ }^{8}$ which justifies the statement by Benedek et al. Hashibon et $a .^{22}$ studied the abrupt, nonmatching $\mathrm{Cu} / \mathrm{Ta}$ interface using first-principles calculations. Relative to the equilibrium $\mathrm{Ta}$ lattice which was taken as the substrate, the misfit strain of the $\mathrm{Cu}(111) / \mathrm{Ta}(110)$ interface is 0.23 and 0.05 in the direction of $[001]_{\mathrm{Ta}}$ and $[110]_{\mathrm{Ta}}$, respectively. They calculated the work of separation and excess interfacial energy of the coherent interfaces with different parallel translation states. By using an energy-weighted averaging method which is essentially similar to the scheme proposed by Benedek et al., ${ }^{4,23}$ they estimated the work of separation and the excess interfacial energy of the commensurate incoherent interface. Their results were shown consistent with the experimental observations. However, the precision of such averaging scheme has not been carefully evaluated.

In the following we first introduce a similar averaging scheme. In principle, starting from the coherent interfacial energies with either Fe or Ag as underlying lattice should yield exactly the same interfacial energy and work of separation for the incoherent interface by using a proper averaging scheme. In the following, for convenience we take $\mathrm{Ag}$ as the underlying lattice, unless otherwise stated.

As suggested in Refs. 4,8,22, and 23, the total work of separation of a semicoherent/incoherent interface may be calculated by averaging the contribution from the individual sites,

$$
W=\bar{W}+\delta W
$$

where

$$
\bar{W}=\frac{1}{n} \sum_{i=1}^{n} W_{i},
$$

with $n$ being the number of Fe atoms at the interface atomic layer and $W_{i}$ is the strain-free work of separation of site $i$, calculated for the coherent interface with the same stacking sequence as the site $i$ at the semicoherent/incoherent interface. 
The "small" quantity $\delta W$ measures the deviation between $\bar{W}$ and the real work of separation of the semicoherent/incoherent interface $W$.

The interfacial energy of the semicoherent/incoherent interface is similarly calculated by averaging over the coherent interfacial energies for interface Fe atoms sitting at different sites at the interface,

$$
\gamma=\bar{\gamma}+\delta \gamma
$$

where

$$
\bar{\gamma}=\frac{1}{n} \sum_{i=1}^{n} \gamma_{i},
$$

with $\gamma_{i}$ being the local interfacial energy contribution from Fe atom at site $i$ at the interface. $\delta \gamma$ measures the small error between $\bar{\gamma}$ and $\gamma$. By definition, $\bar{W}$ and $\bar{\gamma}$ are related by

$$
\bar{W}=\sigma_{\mathrm{Ag}}^{\prime}+\sigma_{\mathrm{Fe}}^{\prime}-\bar{\gamma},
$$

where $\sigma_{\mathrm{Ag}}^{\prime}$ and $\sigma_{\mathrm{Fe}}^{\prime}$ are the strained surface energies of $\mathrm{Ag}$ and $\mathrm{Fe}$. Note that in the present work, the superscript prime symbol (') indicates that the value is calculated at the strained state.

We define the real incoherent work of separation and interfacial energy so that they should be related by the equilibrium surface energies, viz.

$$
W_{\text {incoh }}=\sigma_{\mathrm{Ag}}+\sigma_{\mathrm{Fe}}-\gamma_{\text {incoh }} .
$$

Using Eqs. (6)-(11), for the total error we find

$$
\delta W+\delta \gamma=\left(\sigma_{\mathrm{Fe}}-\sigma_{\mathrm{Fe}}^{\prime}\right)+\left(\sigma_{\mathrm{Ag}}-\sigma_{\mathrm{Ag}}^{\prime}\right) .
$$

When the underlying lattice is that of $\mathrm{Ag}$, the second term from the right-hand side in the above equation vanishes and for the total error we get $0.57 \mathrm{~J} \mathrm{~m}^{-2}$. Unfortunately, we cannot separate the magnitudes of $\delta W$ or $\delta \gamma$ unless we perform explicit large-size supercell calculations for the incoherent interface.

To calculate $\bar{W}$ we need to know $W_{i}$ for all the Fe atoms at different sites. For sites with high symmetry, the work of separations are taken directly from the results for the coherent interfaces. However, for sites away from the high-symmetry positions, people use different ways to evaluate the associated work of separation. Benedek et al. ${ }^{4}$ simply assigned each atom to one of the high-symmetry types. Hashibon et al. ${ }^{22}$ estimated the contribution from an off-symmetry site as a weighted sum of the excess energies of all nearest high symmetry sites with a weight exponentially decaying with the distance from the site. In the present work we explicitly calculated the work of separation for those coherent interfaces with off-symmetry stacking sequences. The work of the separation map for the coherent interface when displacing the bcc Fe part against the fcc Ag part is shown in Fig. 6.

\section{The $\mathrm{Fe}(110) / \mathrm{Ag}(111)$ semicoherent interface}

To estimate the errors ( $\delta W$ and $\delta \gamma$ ) associated with the averaging scheme, we perform direct calculations of the work of separation and interfacial energy of a semicoherent interface. Figure 7 shows the model semicoherent $\mathrm{Fe}(110) / \mathrm{Ag}(111)$ interface with $\mathrm{NW}$ orientation. Only the $\mathrm{Fe}$ and $\mathrm{Ag}$ layers at the interface are shown. Each $\mathrm{Fe}(110)$ layer contains $10 \mathrm{Fe}$ atoms, and each (111) Ag layer contains $8 \mathrm{Ag}$ atoms.

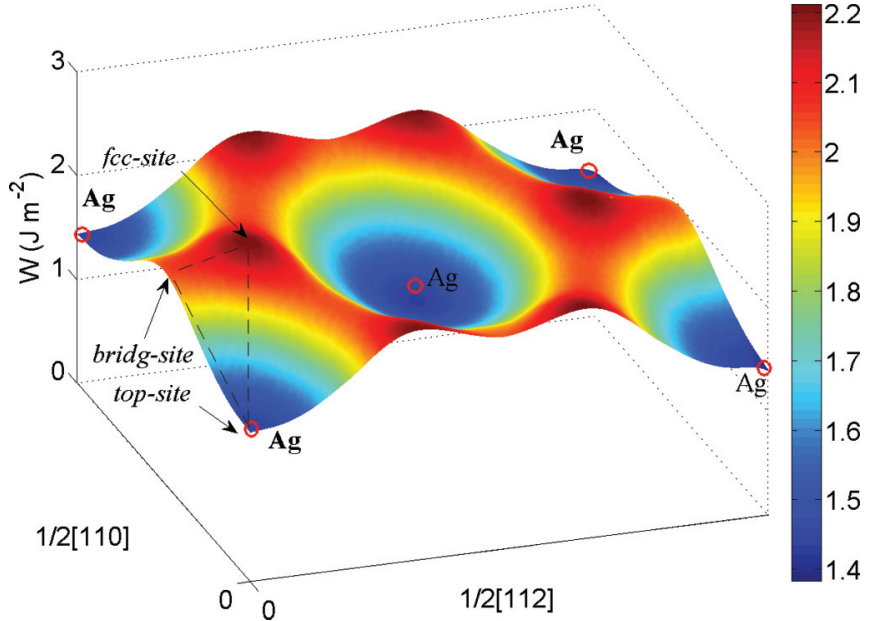

FIG. 6. (Color online) The map of the work of separation obtained by shifting the bcc Fe part above the fcc $\mathrm{Ag}(111)$ plane for the 7-Fe(110)/9- $\mathrm{Ag}(111)$ interface. The $\mathrm{Ag}$ atoms and the high-symmetry positions are marked on the map.

The lattice vectors parallel to the interface are placed to be $[1 \overline{1} 0]_{\mathrm{Ag}} \|[001]_{\mathrm{Fe}}$ and $[1 \overline{1}]_{\mathrm{Ag}} \|[1 \overline{1} 0]_{\mathrm{Fe}}$. Laterally, the Fe lattice is distorted to match the $\mathrm{Ag}$ lattice. The mismatches along the $[001]_{\mathrm{Fe}}$ and $[1 \overline{1} 0]_{\mathrm{Fe}}$ directions are relatively small, $\sim 3.4 \%$ and $\sim 1.4 \%$, respectively. Normal to the interface, all atoms and the lattice constant are relaxed to the equilibrium state. The number of layers are tested and the results show that a $7 \times 7$ supercell (126 atoms) is enough to get a converged work of separation with error less than $0.1 \mathrm{~J} \mathrm{~m}^{-2}$. For this supercell, the work of separation is calculated to be $W_{\text {semicoh }}=2.05 \mathrm{~J} \mathrm{~m}^{-2}$.

In the next step we apply the averaging scheme to evaluate the work of separation of the semicoherent interface in Fig. 7. The averaged work of separation obtained according to Eq. (7) is $\sim 1.91 \mathrm{~J} \mathrm{~m}^{-2}$, which is $\sim 7 \%$ smaller than the result calculated directly for the relaxed supercell $\sim 2.05 \mathrm{~J} \mathrm{~m}^{-2}$ (yielding $\delta W=0.14 \mathrm{~J} \mathrm{~m}^{-2}$ ). Note that the semicoherent interface has the same strain along the $[001]_{\mathrm{Fe}}$ direction as the coherent one,
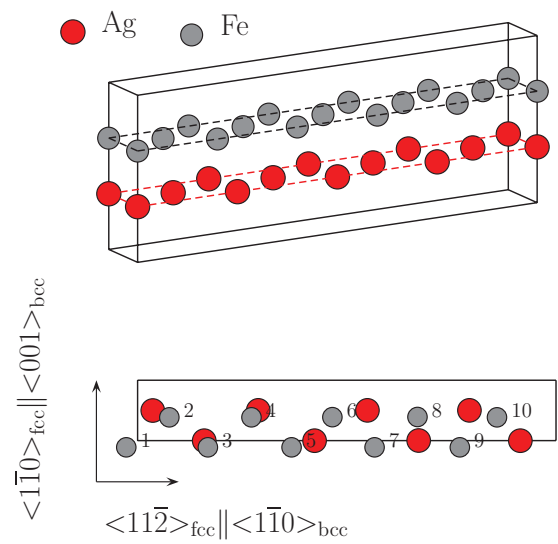

FIG. 7. (Color online) Schematic of the semicoherent interface. Only the Fe and Ag layers at the interface are shown. The upper panel is the side view and the lower panel the top view of the interface. The positions of $\mathrm{Fe}$ atoms at the interface after relaxation are indexed by numbers. 
while along the $[110]_{\mathrm{Fe}}$ direction, it has a much smaller strain $(\sim 3.4 \%)$ than the coherent interface $(\sim 26.8 \%)$. Then from the fact that the strain along this direction does not significantly change the local contribution to the total work of separation, we do not expect the strains along other directions to change the chemical bonding very much, either. There are several findings in literature supporting our conclusion. Wang et al. ${ }^{50}$ reported that the work of separation of the $\mathrm{Al}_{2} \mathrm{O}_{3} / \mathrm{Al}$ interface varies by less than $5 \%$ over the entire range extending from compression of $\mathrm{Al}$ by $4 \%$ to expansion of $\mathrm{Al}_{2} \mathrm{O}_{3}$ by $4 \%$. For the $\mathrm{Si} / \mathrm{Cu}$ interface, they also showed that the work of separation calculated for a fully relaxed $(1 \times 1)$ supercell (containing 1 atom per layer), where both $\mathrm{Cu}$ and $\mathrm{Si}$ lattice are highly distorted, is very close to the accurate value calculated by a very large supercell (overestimated by only $3 \%$ ). ${ }^{51}$

The interfacial energy of the semicoherent interface calculated directly from the supercell method gives $\gamma_{\text {semicoh }}=$ $1.18 \mathrm{~J} \mathrm{~m}^{-2}$, which is significantly larger than the value estimated according to Eq. (9) $\left(\bar{\gamma}=0.65 \mathrm{~J} \mathrm{~m}^{-2}\right)$, so we get $\delta \gamma=0.53 \mathrm{~J} \mathrm{~m}^{-2}$. The significantly smaller interfacial energy from the averaging scheme is due to the presence of magnetism. Magnetism results in a strong dependence of the surface energy and interfacial energy on the in-plane distortion (see Table II). Taking Fe as the underlying lattice, we could avoid distorting the magnetic Fe and thus expect smaller errors with the averaging scheme from the generally larger coherent interfacial energies. Indeed, when taking Fe as the underlying lattice, the averaging scheme generates $\sim 2.00 \mathrm{~J} \mathrm{~m}^{-2}$ for the work of separation and $\sim 0.96 \mathrm{~J} \mathrm{~m}^{-2}$ for the interfacial energy. Both of these figures compare well with the results of the direct supercell calculations which yield $\sim 2.08 \mathrm{~J} \mathrm{~m}^{-2}$ for the work of separation and $\sim 1.10 \mathrm{~J} \mathrm{~m}^{-2}$ for the interfacial energy.

\section{The $\mathrm{Fe}(110) / \mathrm{Ag}(111)$ incoherent interface}

In the present work the commensurate incoherent interface is generated by putting two equilibrium $\mathrm{Fe}$ and $\mathrm{Ag}$ crystals together in the NW orientation relationship without straining any of the lattices. On the 2D interface we define the smallest coincidence site lattice (CSL) with a tolerance of misfit strain of 0.001 . This $2 \mathrm{D}$ repeat cell of the incoherent interface composes of 19 and $29 \mathrm{Fe}$ atoms along the $[110]_{\mathrm{Fe}}$ and $[001]_{\mathrm{Fe}}$ directions, respectively, matching 15 and $28 \mathrm{Ag}$ atoms along the $[11 \overline{2}]_{\mathrm{Ag}}$ and $[1 \overline{1} 0]_{\mathrm{Ag}}$ directions, respectively. In the CSL cell we find some Fe atoms sitting on the fcc-stacking, bridge-stacking, or top-stacking sites, while others are off from these high-symmetry sites.

For the above commensurate $\mathrm{Fe}(110) / \mathrm{Ag}(111)$ incoherent interface, the average work of separation calculated according to Eq. (7) is $\bar{W} \approx 1.86 \mathrm{~J} \mathrm{~m}^{-2}$. Then, according to Eq. (9), we get $\bar{\gamma}=0.70 \mathrm{~J} \mathrm{~m}^{-2}$.

From the results obtained for the semicoherent interface, we could see that both $\delta W$ and $\delta \gamma$ are positive, and $\delta W$ is small and $\delta \gamma$ is large. Therefore, for the incoherent interface when taking $\mathrm{Ag}$ as the underlying lattice, we may expect that $\delta \gamma$ is positive and large, which means that $\bar{\gamma}$ cannot be considered as a good estimation for the incoherent interfacial energy. However, as shown above for the semicoherent interface, $\bar{W}$ may be close to the real incoherent work of separation. We may hence assume that $\bar{W} \approx W_{\text {incoh }}$, and then evaluate the incoherent interfacial energy according to Eq. (11) obtaining $\gamma_{\text {incoh }}=1.27 \mathrm{~J} \mathrm{~m}^{-2}$. In this case we actually assumed that $\delta W$ is zero and the total error $\left(\sigma_{\mathrm{Fe}}-\sigma_{\mathrm{Fe}}^{\prime}\right)$ is imposed on $\bar{\gamma}$.

As shown in the case of semicoherent interface, the significantly smaller $\bar{\gamma}$ is due to the presence of magnetism. If we take $\mathrm{Fe}$ as the underlying lattice and take the corresponding coherent interfacial energies, the resulting total error by the averaging scheme according to Eq. (12), $\delta W+\delta \gamma=\sigma_{\mathrm{Ag}}-$ $\sigma_{\mathrm{Ag}}^{\prime}=0.17 \mathrm{~J} \mathrm{~m}^{-2}$ is much smaller than the one obtained when using $\mathrm{Ag}$ as underlying lattice. In this case, considering that both $\delta W$ and $\delta \gamma$ are positive and they share the total error of $0.17 \mathrm{~J} \mathrm{~m}^{-2}$, we may conclude that both $\bar{W} \approx 1.97 \mathrm{~J} \mathrm{~m}^{-2}$ and $\bar{\gamma} \approx 0.99 \mathrm{~J} \mathrm{~m}^{-2}$ can be considered to be close to the real $W_{\text {incoh }}$ and $\gamma_{\text {incoh}}$.

The resulting work of separation may be considered as the lower bound of the work of separation for the more realistic semicoherent Fe/Ag interface, because such a commensurate interface is not energetically favorable, and upon relaxation it is expected to transfer into a semicoherent interface with periodic coherent areas separated by misfit dislocation arrays. Relaxation decreases the total energy of semicoherent interface $\left(E_{\mathrm{Fe} / \mathrm{Ag}}\right)$ and results in a higher work of separation according to Eq. (2). Correspondingly, the commensurate incoherent interfacial energy may be considered as the upper bound of the interfacial energy for a realistic semicoherent interface.

\section{Interface structure and magnetism}

The interlayer distances at the interface region of the coherent interfaces with different translation states (fcc site, bridge site, and top site) and of the semicoherent interface (see Fig. 7) are shown in Fig. 8(a), taking Ag as the underlying lattice. Note that for the semicoherent interface, the atoms in one layer do not sit exactly in one plane. Therefore, the layer position is calculated by averaging all the atomic positions in that layer and the interlayer distances are calculated according to the averaged layer positions. In Fig. 8 we observe that for the coherent interface with the top-stacking sequence leads to the largest interface separation $\left(d_{11}^{\mathrm{Fe}, \mathrm{Ag}}\right)$. The top site is the least favored position for the $\mathrm{Fe}$ atom, which results in a large separation between $\mathrm{Fe}$ and $\mathrm{Ag}$ layers. For both the bridge-stacking and fcc-stacking sequences, the Fe/Ag interface distance sits in between those of the bulklike $\mathrm{Fe}$ and bulklike Ag. For the semicoherent interface, the average interlayer distance behaves in a similar way as for the coherent interface and the interface separation is intermediate between the largest and the smallest interface separations for the coherent interfaces with different translation states. The interlayer distance for the coherent interfaces with $\mathrm{Fe}$ as the underlying lattice is shown in Fig. 8(b). For both cases we find that larger interlayer distance at interface $\left(d_{11}^{\mathrm{Fe}, \mathrm{Ag}}\right)$ corresponds to smaller work of separation, and vice versa.

The magnetic moments of $\mathrm{Fe}$ atoms near the interface region of the coherent and semicoherent interfaces are shown in Fig. 9. Solid symbols represent the results when taking Ag as the underlying lattice and the open symbols when taking $\mathrm{Fe}$ as the underlying lattice. Magnetic moments of $\mathrm{Fe}$ atoms at the interface for both coherent and semicoherent interfaces are strongly enhanced, due to the weak interaction between $\mathrm{Fe}$ and Ag. ${ }^{45}$ When taking $\mathrm{Ag}$ as the underlying lattice, the 

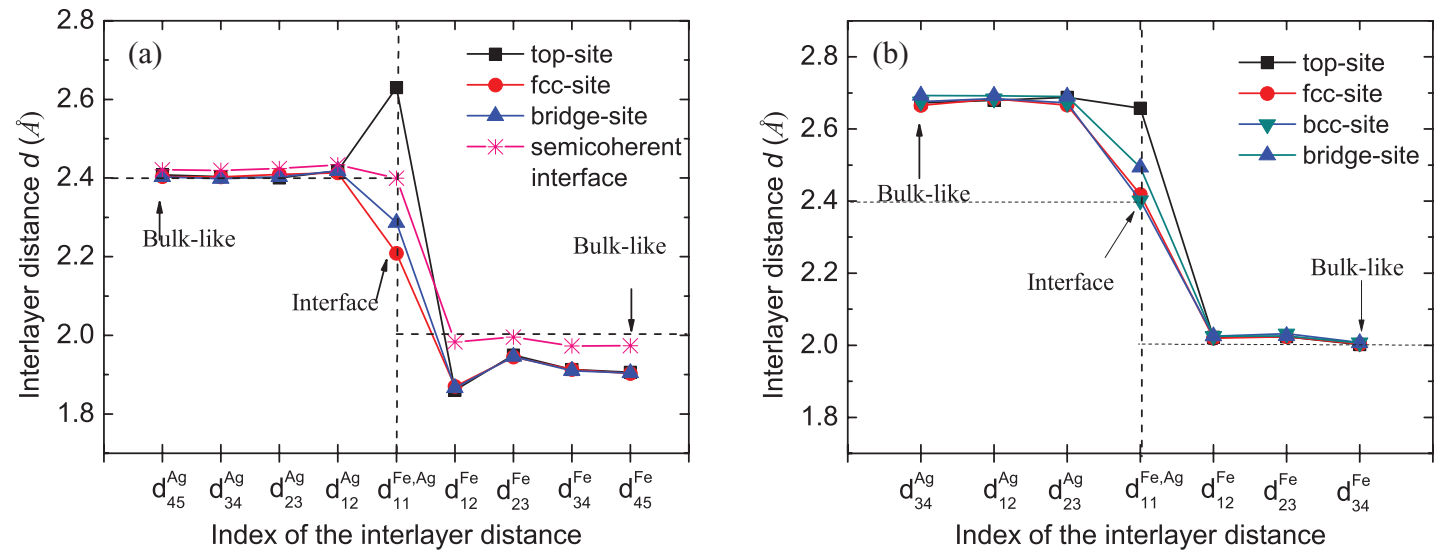

FIG. 8. (Color online) The interlayer distance for the coherent interfaces with different translation states at the interface taking Ag as the underlying lattice (a) and Fe as underlying lattice (b). The interlayer distance for the semicoherent interface is also shown in (a). Notice that the averaged atomic position in one layer was used as the position of the layer in the semicoherent interface.

semicoherent interface has lower magnetic moments for all Fe layers than the coherent interfaces, because the Fe lattice in semicoherent interface is much less distorted. On the other hand, when taking $\mathrm{Fe}$ as the underlying lattice, the interface atom still has a larger magnetic moment compared to the bulk atoms, but they are much smaller than the magnetic moments of the Fe atoms in the distorted Fe lattice. The magnitudes of the magnetic moments for different translation states correlate very well with atomic volume of the $\mathrm{Fe}$ atom at interface, namely, larger atomic volume at interface corresponds to larger magnetic moment. For example, when taking $\mathrm{Ag}$ as the underlying lattice, the interlayer distance has the sequence $d_{11}^{\mathrm{Fe}, \mathrm{Ag}}$ (top site) $>d_{11}^{\mathrm{Fe}, \mathrm{Ag}}$ (bridge site) $>d_{11}^{\mathrm{Fe}, \mathrm{Ag}}$ (fcc site) [Fig. 8(a)], which means that atomic volume of $\mathrm{Fe}$ at the interface $\left(v_{\mathrm{Fe}}\right)$ has the same sequence. Therefore, the magnetic moment of interface $\mathrm{Fe}$ atom is in the same sequence $\mu_{\mathrm{Fe}}$ (top site $)>\mu_{\mathrm{Fe}}$ (bridge site) $>\mu_{\mathrm{Fe}}($ fcc site $)$. The same rule applies for the case of taking $\mathrm{Fe}$ as the underlying lattice.

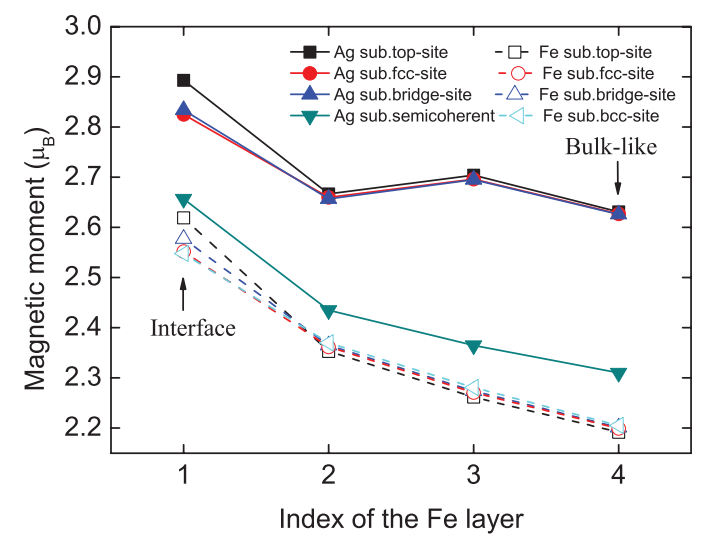

FIG. 9. (Color online) The magnetic moment of the Fe atom with respect to its position relative to the interface for coherent interface and semicoherent interface. The magnetic moment for one layer of the semicoherent interface is averaged over magnetic moments of all the atoms in that layer. Magnetic moments taking $\mathrm{Ag}$ and $\mathrm{Fe}$ as the underlying lattice are represented by solid and open symbols, respectively.
In the case of semicoherent interface, we also monitor the distance $\left(d^{*}\right)$ between each Fe atom within the layer at the interface relative to the averaged position of the conjugated Ag layer at the interface, shown in Fig. 10. The corresponding magnetic moment and atomic volume of $\mathrm{Fe}$ atoms at the interface are shown in Fig. 11. In Fig. 10 we can see that along the $[110]_{\mathrm{Fe}}$ direction $d^{*}$ is wavelike, which means that the $\mathrm{Fe}$ plane at the interface has actually a wavelike atomic surface. The layer puckering was also found at the $\mathrm{MgO} / \mathrm{Cu}$ interface. ${ }^{4}$ The explicit positions of the $\mathrm{Fe}$ atoms after relaxation are shown in Fig. 7. We can see that the Fe atom sitting at/near the top site (fourth $\mathrm{Fe}$ ) has a large $d^{*}$ and the $\mathrm{Fe}$ atom sitting at/near the fcc site (first Fe) has small $d^{*}$. Other Fe atoms sitting at neither of those high-symmetry sites have intermediate $d^{*}$ values. From this point of view, we may justify the assumption that the semicoherent interface is composed of the locally coherent interfaces with different translation states. In Fig. 11 the magnetic moment of $\mathrm{Fe}$ atom at interface also shows a wavelike behavior. The magnitude of the magnetic moment correlates well with the atomic volume, similar to the coherent interfaces. Therefore, there is a spin wave coupled with the

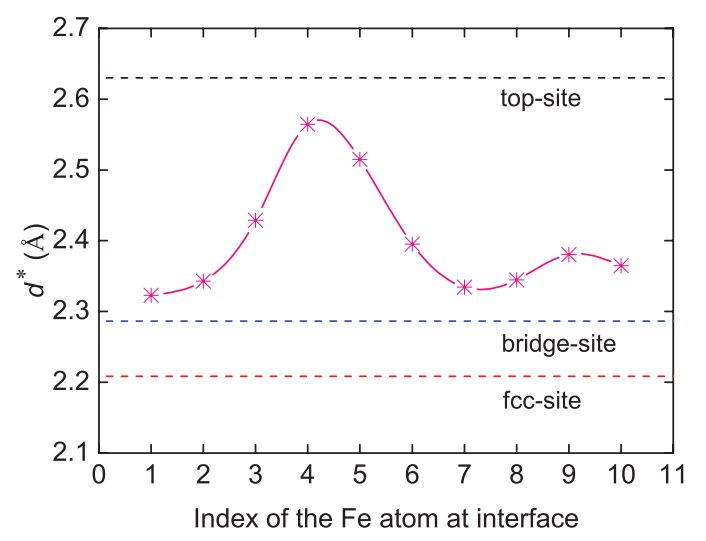

FIG. 10. (Color online) The distance between Fe atoms in the Fe layer at interface relative to the averaged position of the Ag layer at interface. The interface separation for the coherent interfaces with different translation states are marked by dashed lines. See Fig. 7 for the explicit positions of $\mathrm{Fe}$ atoms at interface. 


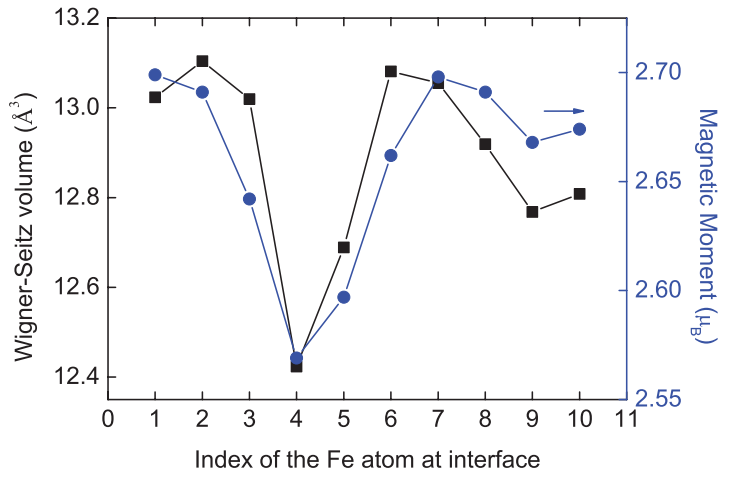

FIG. 11. (Color online) The magnetic moment (right axis) and Wigner-Seitz volume (left axis) for the Fe atoms at the semicoherent interface. See Fig. 7 for the explicit positions of Fe atoms at interface.

puckering Fe layer at the interface. Such complex magnetic behavior near the interface makes any attempt to model the interfaces using bulklike interatomic potentials very difficult and also highly unreliable.

\section{CONCLUSION}

Experimental observations have shown that the real semicoherent $\mathrm{fcc} / \mathrm{bcc}$ interface has various orientation relationships deviating from the Kurdyumov-Sachs (KS) or NW orientation relationships. ${ }^{52,53}$ The actual interface structure is highly dependent on the characteristics of the misfit dislocation arrays. Experimentally, it is very difficult to describe accurately the atomistic structure of the fcc/bcc interface even with today's powerful high-resolution transmission electron microscopy. Without knowing the exact structure of the interface, to accurately determine the interfacial energy theoretically is almost impossible. Actually, the core structure of dislocation and its energy have been a mystery for many years. When misfit dislocations are close, their cores interfere with each other, and then the main contribution to interfacial energy would come from the dislocation core energies which cannot be determined by ab initio calculations at present. However, in the present work, we show that by performing ab initio calculations we can accurately determine the interfacial energy and work of separation of the coherent interface and estimate these energetic parameters for the commensurate incoherent interface. The interfacial energies (work of separation) of the coherent and the incoherent interfaces represent the lower and upper (upper and lower) bounds for the real semicoherent interfaces. By establishing these bounds, we can reasonably assess the contribution to the interfacial energy or work of separation from the misfit dislocations.

Explicitly, we have calculated the work of separation and interfacial energy of the coherent $\mathrm{Fe} / \mathrm{Ag}$ interfaces. These parameters are very important especially when studying the $\mathrm{Fe} / \mathrm{Ag}$ multilayer or the $\mathrm{Fe} / \mathrm{Ag}$ core/shell nanoparticles. For the coherent $\mathrm{Fe}(001) / \mathrm{Ag}(001)$ interface with taking $\mathrm{Ag}$ as the underlying lattice, $W$ and $\gamma$ are calculated to be 2.30 and $0.89 \mathrm{~J} \mathrm{~m}^{-2}$, respectively. For the coherent $\mathrm{Fe}(110) / \mathrm{Ag}(111)$ interface, taking $\mathrm{Ag}$ or $\mathrm{Fe}$ as the underlying lattice, the largest $W$ and smallest $\gamma$ are calculated to be 2.21 and $0.36 \mathrm{~J} \mathrm{~m}^{-2}$ or 2.24 and $0.79 \mathrm{~J} \mathrm{~m}^{-2}$, respectively. The significant difference in the interfacial energy when using different underlying lattices is due to the magnetism. We propose an averaging scheme to estimate the work of separation and interfacial energy for the semicoherent/commensurate incoherent interface. We justify the use of the averaging scheme by performing large supercell calculations for a semicoherent interface. We have shown that taking either $\mathrm{Fe}$ or $\mathrm{Ag}$ as the underlying lattice, the averaging scheme can yield a reasonable estimation of the work of separation of the semicoherent interface. However, when taking $\mathrm{Ag}$ as the underlying lattice the averaged interfacial energy of the semicoherent interface is significantly underestimated due to the magnetism. When taking $\mathrm{Ag}(\mathrm{Fe})$ as the underlying lattice, applying the averaging scheme we get $1.86(1.96) \mathrm{J} \mathrm{m}^{-2}$ for the work of separation and $1.27(0.99)$ $\mathrm{J} \mathrm{m}^{-2}$ for the interfacial energy of the incoherent interface. We study the crystal and magnetic structures at the coherent and semicoherent interfaces and show that at a real semicoherent interface the atomic plane is wavelike and there is spin wave coupled with the interface Fe layer.

\section{ACKNOWLEDGMENTS}

Authors acknowledge the Swedish Research Council, the European Research Council (Grant No. 228074), the Swedish Foundation for International Cooperation in Research and Higher Education, the Hungarian Scientific Research Fund (research project OTKA 84078), the Swedish Steel Producer's Association, the Carl Tryggers Foundation, the Emil Aaltonen foundation and the China Scholarship Council for financial support. National supercomputer centre in Linköping Sweden and the Finnish IT Center for Science are acknowledged for supplying computational resource.
*Corresponding author: lusommmg@ hotmail.com

${ }^{1}$ E. Kozeschnik, Scr. Mater. 59, 1018 (2008).

${ }^{2}$ R. Shi, N. Ma, and Y. Wang, Acta Mater. 60, 4172 (2012).

${ }^{3}$ Y. Wang and J. Li, Acta Mater. 58, 1212 (2010).

${ }^{4}$ R. Benedek, A. Alavi, D. N. Seidman, L. H. Yang, D. A. Muller, and C. Woodward, Phys. Rev. Lett. 84, 3362 (2000).

${ }^{5}$ T. Fujii, H. Nakazawa, M. Kato, and U. Dahmen, Acta Mater. 48, 1033 (2000).

${ }^{6}$ J. Rigsbee and H. Aaronson, Acta Metall. 27, 351 (1979).
${ }^{7}$ M. Hall, H. Aaronson, and K. Kinsma, Surf. Sci. 31, 257 (1972).

${ }^{8}$ S. A. E. Johansson, M. Christensen, and G. Wahnström, Phys. Rev. Lett. 95, 226108 (2005).

${ }^{9}$ D. Watanabe, C. Watanabe, and R. Monzen, J. Mater. Sci. 43, 3946 (2008).

${ }^{10}$ Y. Yao and T. Wang, Acta Mater. 47, 3063 (1999).

${ }^{11}$ Y. Yao, T. Wang, and C. Wang, Phys. Rev. B 59, 8232 (1999).

${ }^{12}$ J. Raynolds, E. Roddick, J. Smith, and D. Srolovitz, Acta Mater. 47, 3281 (1999). 
${ }^{13}$ N. Medvedeva, Y. Gornostyrev, O. Kontsevoi, and A. Freeman, Acta Mater. 52, 675 (2004).

${ }^{14}$ X. Gu and W. Zhang, Solid State Phenom. 172-174, 260 (2011).

${ }^{15}$ A. Hashibon, P. Schravendijk, C. Elsässer, and P. Gumbsch, Philos. Mag. 89, 3413 (2009).

${ }^{16}$ J. K. Chen, D. Farkas, and W. T. Reynolds Jr., Acta Mater. 45, 4415 (1997).

${ }^{17}$ Z. Yang and R. A. Johnson, Model. Simul. Mater. Sci. Eng. 1, 707 (1993).

${ }^{18}$ Y. Mishin, Handbook of Materials Modeling, edited by S. Yip (Springer, Dordrecht, The Netherlands, 2005), Chap. 2.2, p. 22.

${ }^{19}$ A. Hashibon, A. Y. Lozovoi, Y. Mishin, C. Elsässer, and P. Gumbsch, Phys. Rev. B 77, 094131 (2008).

${ }^{20} \mathrm{~S}$. Hu, M. Baskes, M. Stan, and L. Chen, Acta Mater. 54, 4699 (2006).

${ }^{21}$ V. Vaithyanathan, Ph.D. thesis, Pennsylvania State University, 2002.

${ }^{22}$ A. Hashibon, C. Elsässer, Y. Mishin, and P. Gumbsch, Phys. Rev. B 76, 245434 (2007).

${ }^{23}$ R. Benedek, D. N. Seidman, and C. Woodward, J. Phys.: Condens. Matter 14, 2877 (2002).

${ }^{24}$ N. C. Koon, B. T. Jonker, F. A. Volkening, J. J. Krebs, and G. A. Prinz, Phys. Rev. Lett. 59, 2463 (1987).

${ }^{25}$ L. Lu, W. Zhang, D. Wang, X. Xu, J. Miao, and Y. Jiang, Mater. Lett. 64, 1732 (2010).

${ }^{26}$ K. J. Carroll, D. M. Hudgins, S. Spurgeon, K. M. Kemner, B. Mishra, M. I. Boyanov, L. W. Brown, M. L. Taheri, and E. E. Carpenter, Chem. Mater. 22, 6291 (2010).

${ }^{27}$ G. Smith, H. Padmore, and C. Norris, Surf. Sci. Lett. 119, L287 (1982).

${ }^{28}$ B. Jonker and G. Prinz, Surf. Sci. Lett. 172, L568 (1986).

${ }^{29}$ M. Benoit, C. Langlois, N. Combe, H. Tang, and M.-J. Casanove, Phys. Rev. B 86, 075460 (2012).

${ }^{30}$ C. Borchers and R. Bormann, Acta Mater. 53, 3695 (2005).
${ }^{31}$ A. Hashibon, C. Elsässer, and M. Rhle, Acta Mater. 53, 5323 (2005).

${ }^{32}$ P. Hohenberg and W. Kohn, Phys. Rev. 136, B864 (1964).

${ }^{33}$ G. Kresse and J. Hafner, Phys. Rev. B 47, 558 (1993).

${ }^{34}$ G. Kresse and J. Furthmüller, Phys. Rev. B 54, 11169 (1996).

${ }^{35}$ G. Kresse and D. Joubert, Phys. Rev. B 59, 1758 (1999).

${ }^{36}$ J. P. Perdew, K. Burke, and M. Ernzerhof, Phys. Rev. Lett. 77, 3865 (1996).

${ }^{37}$ P. E. Blöchl, Phys. Rev. B 50, 17953 (1994).

${ }^{38}$ Y. Wang, W. Wang, K.-N. Fan, and J. Deng, Surf. Sci. 490, 125 (2001).

${ }^{39}$ H. Fu, L. Jia, W. Wang, and K. Fan, Surf. Sci. 584, 187 (2005).

${ }^{40}$ M. Methfessel, D. Hennig, and M. Scheffler, Phys. Rev. B 46, 4816 (1992).

${ }^{41}$ H. Erschbaumer, A. Freeman, C. Fu, and R. Podloucky, Surf. Sci. 243, 317 (1991).

${ }^{42}$ E. Krotscheck and W. Kohn, Phys. Rev. Lett. 57, 862 (1986).

${ }^{43}$ W. Tyson and W. Miller, Surf. Sci. 62, 267 (1977).

${ }^{44}$ M. P. J. Punkkinen, S. K. Kwon, J. Kollár, B. Johansson, and L. Vitos, Phys. Rev. Lett. 106, 057202 (2011).

${ }^{45}$ C. L. Fu, A. J. Freeman, and T. Oguchi, Phys. Rev. Lett. 54, 2700 (1985).

${ }^{46}$ R. Richter, J. G. Gay, and J. R. Smith, Phys. Rev. Lett. 54, 2704 (1985).

${ }^{47}$ S. I. Popel, V. N. Kozhurkov, and A. A. Zhukov, Russ. Metal. 5, 56 (1975).

${ }^{48}$ F. Spaepen, Mater. Sci. Eng. A 178, 15 (1994).

${ }^{49}$ K. Kita and R. Monzen, J. Jpn. Inst. Met. 65, 223 (2001).

${ }^{50}$ X.-G. Wang, J. R. Smith, and A. Evans, Phys. Rev. Lett. 89, 286102 (2002).

${ }^{51}$ X.-G. Wang and J. R. Smith, Phys. Rev. Lett. 95, 156102 (2005).

${ }^{52}$ C. Luo, U. Dahmen, and K. Westmacott, Acta Metall. Mater. 42, 1923 (1994).

${ }^{53}$ H. Jiao, M. Aindow, and R. C. Pond, Philos. Mag. 83, 1867 (2003). 